Vol. 45 (1992) [151-155]

\title{
A NOTE ON HIGH DEGREE LINEAR COMPLEMENTARITY PROBLEMS
}

\author{
David E Stewart
}

\begin{abstract}
Topological degree theory can be applied to maps defined from Linear Complementarity Problems, as has been done by Howe and Stone, Ha, and Stewart. It is shown here that the definitions of Howe and Stone, and Stewart, are equivalent. Also a new family of matrices is defined whose degrees' magnitudes increase exponentially as $2^{n} / \sqrt{2 \pi n}$, whereas Howe and Stone give examples whose degrees go as $\left(2^{2 / 5}\right)^{n}$.
\end{abstract}

\section{Degree of LCPS}

A Linear Complementarity Problem (LCP) is a problem where a square matrix $M \in \mathbf{R}^{n \times n}$ and a vector $q \in \mathbf{R}^{n}$ are given and we wish to find vectors $z, w \in \mathbf{R}^{n}$ where

$$
w=M z+q \geqslant 0, \quad z \geqslant 0, \quad w^{T} z=0 .
$$

(The inequalities are understood to hold componentwise.) This problem is denoted $\operatorname{LCP}(M, q)$.

The matrix $M$ is said to be nondegenerate if it has no singular principal submatrices. It is said to be weakly nondegenerate if $\operatorname{LCP}(M, 0)$ has only the trivial solution $(z=w=0)$. A solution $(z, w)$ is said to be nondegenerate if for each $i=1, \ldots, n$, either $z_{i}>0$ or $w_{i}>0$. It is well known that if $M$ is nondegenerate then every solution of $\operatorname{LCP}(M, q)$ is nondegenerate for almost all $q$ [3].

An LCP can be associated with families of nonlinear maps $\mathbf{R}^{n} \rightarrow \mathbf{R}^{n}$. These maps have an associated topological degree, and so we can define a "degree" for the problem $\operatorname{LCP}(M, q)$; if $M$ is weakly nondegenerate, then this degree is independent of $q$. This degree is of importance because if a matrix $M$ has "LCP degree" $d$, then $\operatorname{LCP}(M, q)$ has at least $|d|$ solutions if they are all nondegenerate.

There is more than one way to choose a suitable nonlinear map $\mathbf{R}^{\mathbf{n}} \rightarrow \mathbf{R}^{\mathbf{n}}$. In [4] the map $F_{M, q}(z)=\min (z, M z+q)$ is used; in [2] the map $P_{M}(x)=(x+|x|) / 2-$ $M(|x|-x) / 2$ is used. In the former case we wish to solve $F_{M, q}(z)=0$ and the associated solution of $\operatorname{LCP}(M, q)$ is $(z, M z+q)$; in the latter case the equation to be solved

Received 15 February 1991

Copyright Clearance Centre, Inc. Serial-fee code: 0004-9729/92 \$A2.00+0.00. 
is $P_{M}(x)=q$ and the solution associated is $\left((-x)_{+}, x_{+}\right)$. The degrees of $\operatorname{LCP}(M, q)$ are defined respectively as $\operatorname{deg} F_{M, q}=\operatorname{deg}\left(F_{M, q}, r_{0} B, 0\right)$ where $B$ is the unit ball, and $r_{0}$ is sufficiently large so that all solutions of $\operatorname{LCP}(M, q)$ satisfy $\|z\|<r_{0}$; and for the second map, the degree is defined as the topological degree of $\Pi_{M}: S^{n-1} \rightarrow S^{n-1}$ where $\Pi_{M}(x)=P_{M}(x) /\left\|P_{M}(x)\right\|$. (Here $S^{n-1}$ denotes the unit sphere in $\mathrm{R}^{n}$.)

Both of these degrees are defined for weakly nondegenerate matrices $M$ and all $q$. That the degree of $\operatorname{LCP}(M, q)$ depends only on $M$ in the former case if $M$ is weakly nondegenerate can be easily seen as we can use the homotopy invariance of the degree; at no point in a homotopy with only $q$ varying do the solutions become unbounded.

The two degrees in fact turn out to be the same. If $M$ is nondegenerate then, for almost all $q$, all the solutions of $\operatorname{LCP}(M, q)$ are nondegenerate. In such a case the degree of $F_{M, q}$ and $\Pi_{M}$ are both equal to the sum of the indexes of the solutions $F_{M, q}(z)=0$ and $P_{M}(x)=q$ respectively. The index of a solution $(z, w)$ (whether defined in terms of either $z$ or $x)$ is sgn det $M_{I I}$ where $I=\left\{i \mid z_{i}>0\right\}$, whichever degree is used. (Here $M_{I I}$ denotes the $|I| \times|I|$ matrix

$$
\left[\begin{array}{cccc}
m_{i_{1} i_{1}} & m_{i_{1} i_{2}} & \ldots & m_{i_{1} i_{k}} \\
m_{i_{2} i_{1}} & m_{i_{2} i_{2}} & \ldots & m_{i_{2} i_{k}} \\
\vdots & \vdots & \ddots & \vdots \\
m_{i_{k} i_{1}} & m_{i_{k} i_{2}} & \ldots & m_{i_{k} i_{k}}
\end{array}\right]
$$

where $I=\left\{i_{1}, i_{2}, \ldots, i_{k}\right\}$ and $i_{1}<i_{2}<\cdots<i_{k}$.) As the indexes for the two mappings are the same for each solution, it follows that the degrees of the two mappings must be the same for all nondegenerate matrices. This can be extended to all weakly nondegenerate matrices by a homotopy argument along with the fact that the set of weakly nondegenerate $n \times n$ matrices forms an open set.

As the degrees of $F_{M, q}$ and $\Pi_{M}$ are equal, this common degree will be referred to as the "LCP degree" of $M$, denoted "LCP $\operatorname{deg} M$ ". As the degree of a mapping can be used as a measure of its topological complexity, it is interesting to discover matrices whose degree is large. It should also be noted that the most common algorithms for solving LCPs only work for matrices whose LCP degree is \pm 1 . Indeed, the class of strictly copositive matrices (where $u \geqslant 0, u \neq 0$ implies $u^{T} M u>0$ ), which can be solved by Lemke's algorithm have LCP degree +1 . High LCP degree matrices can be difficult to handle computationally.

\section{A FAMILY OF HIGH DEGREE LCPS}

It has been shown by Howe and Stone [2] that the LCP degree of a matrix can be at most exponentially large. In particular, they show the following: 
Theorem 2.1. If $M \in \mathrm{R}^{n \times n}$ is nondegenerate then

$$
|\mathrm{LCP} \operatorname{deg} M| \leqslant \frac{3}{8} 2^{n}-1 \text {. }
$$

(See [2, Proposition 5.1].)

Further they provide a family of examples $M_{n} \in \mathbf{R}^{n \times n}$ where

$$
\operatorname{deg} M_{n} \geqslant 2^{(2 / 5) n-1} \text {. }
$$

(See [2, Proposition 5.3].)

The main result of this note follows:

TheOREM 2.2. There are $n \times n$ matrices $M_{n}$ whose LCP degree has magnitude at least as large as

$$
\frac{1}{2}\left(\begin{array}{c}
n \\
\lceil n / 2\rceil
\end{array}\right)
$$

ProOF: The proof consists of showing that the $n \times n$ matrix

$$
M=\left[\begin{array}{ccccc}
5 / 2-n & 2 & 2 & \ldots & 2 \\
2 & 5 / 2-n & 2 & \ldots & 2 \\
2 & 2 & 5 / 2-n & \ldots & 2 \\
\vdots & \vdots & \vdots & \ddots & \vdots \\
2 & 2 & 2 & \ldots & 5 / 2-n
\end{array}\right]
$$

has degree with magnitude greater than $\left(\begin{array}{c}n \\ {[n / 2]}\end{array}\right) / 2$.

It is easy to check that this matrix is nondegenerate using the formula

$$
\operatorname{det}\left[\begin{array}{cccc}
-\alpha & \beta & \ldots & \beta \\
\beta & -\alpha & \ldots & \beta \\
\vdots & \vdots & \ddots & \vdots \\
\beta & \beta & \ldots & -\alpha
\end{array}\right]=(-1)^{n}((n-1) \beta-\alpha)(\alpha+\beta)^{n-1}
$$

We consider the problem $\operatorname{LCP}(M,-e)$ where $e=[1,1, \ldots, 1]^{T}$. As $-e$ is not a linear combination of $(n-1)$ columns of $M$, every solution of $\operatorname{LCP}(M,-e)$ is nondegenerate. For each solution $(z, w)$ of $\operatorname{LCP}(M,-e)$ we let $I=\left\{i \mid z_{i}>0\right\}$. By symmetry and uniqueness of the solution with a given "active set" $I$, it follows that $z_{i}$ is constant for all $i \in I$, and zero otherwise. Let $\rho_{I}$ be this constant.

Writing $M=\beta e e^{T}-(\alpha+\beta) I$ we see that $w=M z-e$ becomes $w_{i}=\beta \rho_{I}|I|-$ $(\alpha+\beta) \rho_{I}-1$ for $i \in I$ and $w_{i}=\beta \rho_{I}|I|-1$ otherwise. As $z_{i}>0$ for $i \in I$, we have 
$w_{i}=0$ for $i \in I$; this implies that $((|I|-1) \beta-\alpha) \rho_{I}=1$. Thus $\rho_{I}>0$ if and only if $(|I|-1) \beta>\alpha$. To have $w_{i}>0$ for $i \notin I$, we need $\alpha+\beta>0$.

For this solution $(z, w)$ of $\operatorname{LCP}(M,-e)$, the index is

$$
\begin{aligned}
\operatorname{sgn} \operatorname{det} M_{I I} & =\operatorname{sgn}(-1)^{|I|}((|I|-1) \beta-\alpha)(\alpha+\beta)^{|I|-1} \\
& =(-1)^{|I|} .
\end{aligned}
$$

Choosing $\alpha, \beta>0$ we have solutions $(z, w)$ with active set $I$ for any subset $I$ of $\{1, \ldots, n\}$ provided $|I|>1+(\alpha / \beta)$. Hence

$$
L C P \operatorname{deg} M=\sum_{k=1+\lceil\alpha / \beta\rceil}^{n}(-1)^{k}\left(\begin{array}{l}
n \\
k
\end{array}\right) .
$$

Now it is a matter of simplifying this sum to obtain the result.

For the values of $\alpha$ and $\beta$ chosen at the beginning of the proof, $1+\lceil\alpha / \beta\rceil=\lceil n / 2\rceil$. If $n=2 m$ ( $n$ even), then

$$
\text { LCP } \operatorname{deg} M=\frac{1}{2}(-1)^{m+1}\left(\begin{array}{c}
2 m \\
m
\end{array}\right)
$$

and if $n=2 m+1$ ( $n$ odd), then

$$
\text { LCP } \operatorname{deg} M=\frac{1}{2}(-1)^{m+1} \frac{2 m+2}{2 m+1}\left(\begin{array}{c}
2 m+1 \\
m+1
\end{array}\right) \text {. }
$$

Clearly in both cases $\mid$ LCP $\operatorname{deg} M \mid \geqslant \frac{1}{2}\left(\begin{array}{c}n \\ {[n / 2]}\end{array}\right)$ as required (in fact, strictly greater if $n$ is odd).

Asymptotically, this lower bound on the maximum LCP degree of an $n \times n$ matrix is $2^{n} / \sqrt{2 \pi n}$ by Stirling's approximation of the factorial function. This gives a somewhat sharper bound on the rate of growth of the maximum LCP degree of a matrix than in [2]: Now we have

$$
\frac{\ln \max \left\{|\mathrm{LCP} \operatorname{deg} M| \mid M \in \mathbf{R}^{n \times n}\right\}}{n} \sim \ln 2 \quad \text { as } n \rightarrow \infty .
$$

A question remains: Does $\max \left\{|\mathrm{LCP} \operatorname{deg} M| \mid M \in \mathbf{R}^{n \times n}\right\} \geqslant C 2^{n}$ for some constant $C>0$ and sufficiently large $n$ ?

ADDED IN PROOF. It has recently come to the author's attention that the results of this paper have been improved by geometric techniques in ' $O$ n the maximum degree of an LCP map' by Walter Morris, Jr., Math. Oper. Res., 15 (1990) 423-429. Morris found $n \times n$ matrices $M_{n}$ with $\left|\operatorname{LCP} \operatorname{deg} M_{n}\right|=\left(\begin{array}{c}n-1 \\ (n-1) / 2\end{array}\right)$. This agrees with the above lower bounds for the LCP degree of $n \times n$ matrices for $n$ even, but is better for $n$ odd by a factor of about $1+1 / n$. 


\section{REFERENCES}

[1] Ha, C.D., 'Application of degree theory in stability of the complementarity problem', Math. Oper. Res. 12 (1987), 368-376.

[2] R. Howe and R. Stone, 'Linear complementarity and the degree of mappings', in Homotopy methods and global convergence, Editors B.C. Eaves, F.J. Gould, H.-O. Peitgen and M.J. Todd, pp. 179-223 (Plenum Press, New York, London).

[3] K. Murty, 'On the number of solutions to the complementarity problem and spanning properties of complementary cones', Linear Algebra Appl. 5 (1972), 65-108.

[4] D.E. Stewart, 'A degree theory approach to degeneracy of LCPs', Linear Algebra Appl. (submited).

Statistics Department

School of Mathematical Sciences

Australian National University

GPO Box 4

Canberra ACT 2601

Australia 